\title{
Design Scheme of Communication Node Based on FlexRay Bus
}

\author{
Xin $\mathrm{He}^{1}$, Jia'nan $\mathrm{Wu}^{1}$, Hongde Deng ${ }^{1}$, Zean Zhen ${ }^{1}$ and Chenyang Liu ${ }^{1}$ \\ ${ }^{1}$ School of Automation, Northwestern Polytechnical University, China
}

\begin{abstract}
As a high-speed and reliable serial communication bus, the FlexRay bus has gradually become the mainstream of vehicle network systems. In order to verify the feasibility of the bus technology in aerospace applications, this paper designs a FlexRay communication node based on TI's high-performance microcontroller TMS570LS3137 for security systems. In the whole system, with the DSP of core, the FlexRay communication protocol is achieved by using the bus controller TJA1080 to carry out data transmission. This method is suitable for data transmission of pint and medium-sized UAV (unmanned air vehicles) flight control system with high integration and high transmission rate.
\end{abstract}

\section{Introduction}

Aviation bus [1-2] is one of the basic and key technologies of avionics system. With the development of airborne avionics technology, higher requirements are proposed for the airborne data bus. At present, the data bus is widely used on modern flight control computers to realize data exchange and sharing among its various subsystems. While improving the reliability, expansibility and flexibility of the airborne electronic system, the bus performance indirectly determines the flight performance and flight safety of UAV (unmanned air vehicles). Therefore, it is an important means for the research on bus technology to improve the performance of the flight control system.

Currently, among the communication bus widely used in the aviation field, the communication rates of the 1553B, ARINC659, and other buses all meet the requirements, but the cost is too high. The transmission rate of the ARINC429 bus is only $100 \mathrm{Kbps}$ [3-4], which is not suitable to use in pint and medium-sized UAV. The CAN bus has been widely used due to its reliable performance and strong anti-interference ability, and the real-time and reliability of data communication have also been fully verified [5]. However, with the rapid development of new type UAV, the CAN bus cannot meet the index requirements of increasingly complex system, due to its limitations of communication rate and communication mechanism. Thus, it is necessary to find a new bus technology with high data transmission rate and high security to meet the large-capacity and real-time communication requirements of new type UAV flight control system.

The new generation of vehicle bus FlexRay [6-7] has high transmission rate, high reliability, high security and low cost, which data transmission rate up to $10 \mathrm{Mbps}$, efficiency up to $96.9 \%$. As its performance is not lower than other mainstream aviation bus, it is an inevitable trend to adopt it as the communication network of new type UAV. This paper designs a communication node for pint and medium-sized UAV flight control system, which based on the technology of FlexRay bus.

\section{Design of FlexRay bus node}

\subsection{Node architecture}

There are three kinds of architecture modes for FlexRay node: MCU (microprocessor), CC (communication controller) and BD (bus driver), MCU (microprocessor) with $\mathrm{CC}$ (communication controller) built-in and BD (bus driver), MCU (microprocessor) with CC (communication controller) and BD (bus driver) built-in. The current commonly used are MCU + CC + BD and MCU + BD [8-9]. This system adopts MCU + BD structure, as shown in Fig. 1, among them, the MCU adopts TI Company's TMS570LS3137, and the bus driver adopts NXP Company's TJA1080.

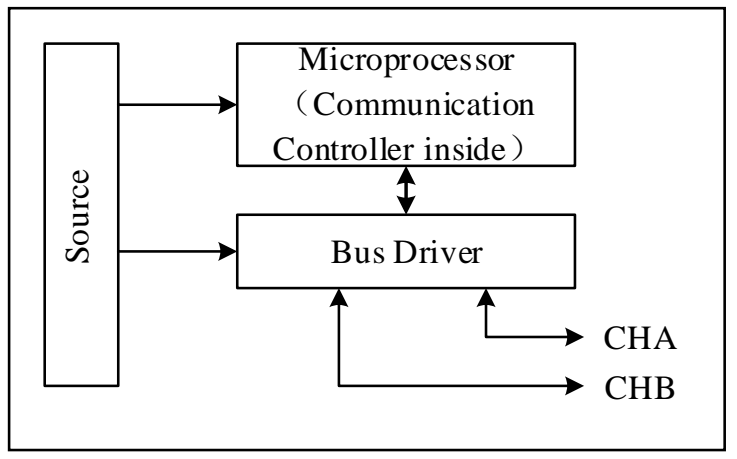

Figure 1. MCU+BD Structure. 


\subsection{Microprocessor selection}

According to the demand of the bus node, it has great influence on security in system. The Hercules series in TI (Texas Instrument), can meet this requirements, which include three kinds of MCU (RM4x, TMS570 and TMS470M) based on ARM Cortex. Among them, the TMS570 is the most suitable for the aerospace fields, railway signal process, etc. At last, TMS570LS3137PGE is selected in this system.

\subsection{Design of the whole system structure}

The MCU sets its working status by configuring the relevant registers in the communication controller, and realizes the FlexRay bus data transmission by setting the FlexRay protocol status. The bus driver connects the communication controller and the FlexRay bus, and the relevant data in the communication controller is transferred to the bus through the encoding mechanism, and the received data on the bus transmitted to the communication controller through the decoding mechanism. Then, the FlexRay bus communication is realized.

As a node on the FlexRay bus, this system sends signals to the bus and receives the signals transmitted on the bus, and then the related communication tasks are completed. The main task of the FlexRay bus node is data transmission. The whole system consists of TMS570LS3137, TJA1080, SCI and JTAG, etc, as shown in Fig. 2.

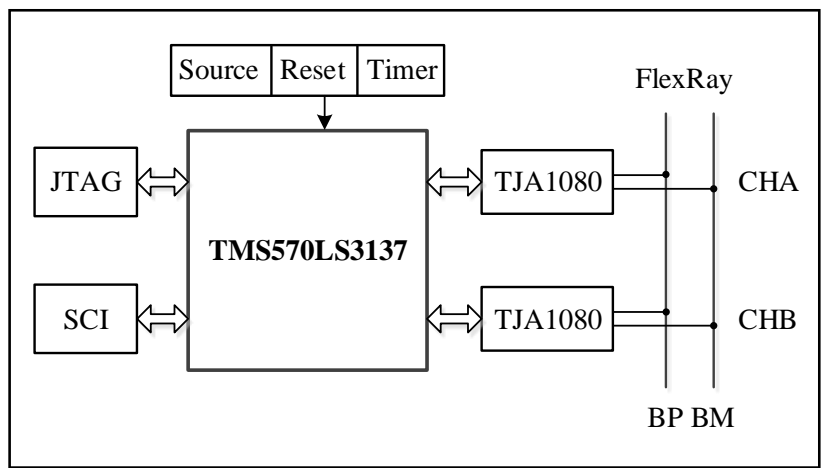

Figure 2. Structure of the Whole System.

\section{Key devices}

\subsection{TJA1080}

TJA1080 is a FlexRay bus driver compliant with the FlexRay protocol version 2.1. It is designed to meet the high-speed communication system from $1 \mathrm{Mb}$ to $10 \mathrm{Mb}$, and is optimized for time-triggered communication protocols, which is to provide the physical interfaces between modules and channels, and provide differential transmit and receive. It is very flexible and can be used as a node transceiver or an active star transceiver, with excellent ESD (electro-static discharge) protection and power management capabilities [10]. In node mode, the TJA1080 transceiver operates as a single device. In star mode, the transceiver acts as a branch of the FlexRay active star network.

In the power-up stage, if the TRxD0 and TRxD1 are grounded, the transceiver can be configured in a node mode and the following operating modes are selectable:

- Normal (normal power mode)

- Receive-only (normal power mode)

- Standby (low power mode)

- Go-to-sleep (low power mode)

- Sleep (low power mode)

In the power-up stage, if the TRxD0 and TRxD1 are connected to the VBUF, the transceiver can be configured in a star mode and the following operating modes are available:

- Star-idle (normal power mode)

- Star-transmit (normal power mode)

- Star-receive (normal power mode)

- Star-sleep (low power mode)

- Star-standby (low power mode)

- Star-locked (normal power mode)

This paper set it to node mode. Under node configuration, TJA1080 provides two control pins EN and STBN to select the operating mode, its pin function is as shown in the Fig. 3.

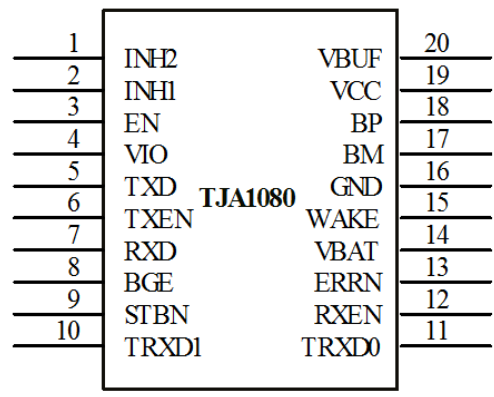

Figure 3. Pin Configuration.

\subsection{ACT1210}

The essence of the FlexRay signal is a differential signal, so the common mode signal on the bus is the interference signal. Therefore, it is necessary to add a common mode choke in the design. The two coils of the common mode choke are wound on the same core with the same number of turns and the reversed twining direction. In this way, as the differential mode current passes through the common mode choke, the current generates reverse magnetic field in the coil and cancel each other out. And when the common mode current passes through the common mode choke, a co-directional magnetic field is generated in the coil to increase the inductance of the coil, then the coil for common mode signal is characterized by high impedance state, and hinder the common mode signal.

Actually, it is equivalent to a bi-directional filter. On the one hand, it eliminates the common mode electromagnetic interference transmitted over the bus. On the other hand, it controls its own circuit not radiate electromagnetic waves to the outside, to avoid affecting other electronic devices. Common mode choke can transmit differential mode signals, even the signals of direct current and very low frequency can pass. However, 
it exhibits high impedance for high-frequency common mode noise, and it can be used to inhibit the common mode interference, reduce the electromagnetic radiation, and enhance the EMC (electromagnetic compatibility) of the circuit [10].

The common mode choke in this paper is TDK Company's ACT1210, which is common to the FlexRay bus and CAN bus. It is the same as the winding common mode choke, but realizing the miniaturization. The appearance and its internal circuit are shown in the Fig. 4.
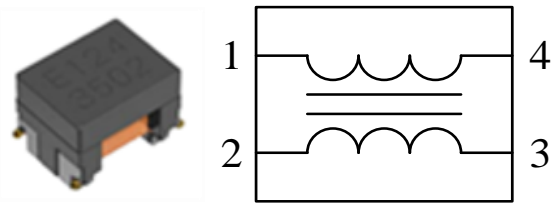

Figure 4. ACT1210.

\section{Driving circuit design}

The connection between TMS570LS3137 and TJA1080 is the main component of the system hardware design, which is the bridge between the microprocessor and the FlexRay bus. The TMS570LS3137 integrates dualchannel FlexRay bus communication controllers and $8 \mathrm{~KB}$ message RAM, and the FlexRay controller uses a dualchannel serial, fixed time base multi-master communication protocol with communication rate of $10 \mathrm{Mbps}$ per channel.

The driving circuit is a bridge connecting the MCU and the FlexRay bus node, which transmits instruction to the FlexRay bus node. Moreover, the filtering isolation circuit is added before the bus node, which increases the stability and anti-interference ability during the data transmission. The circuit schematic diagram is shown in the Fig. 5 .

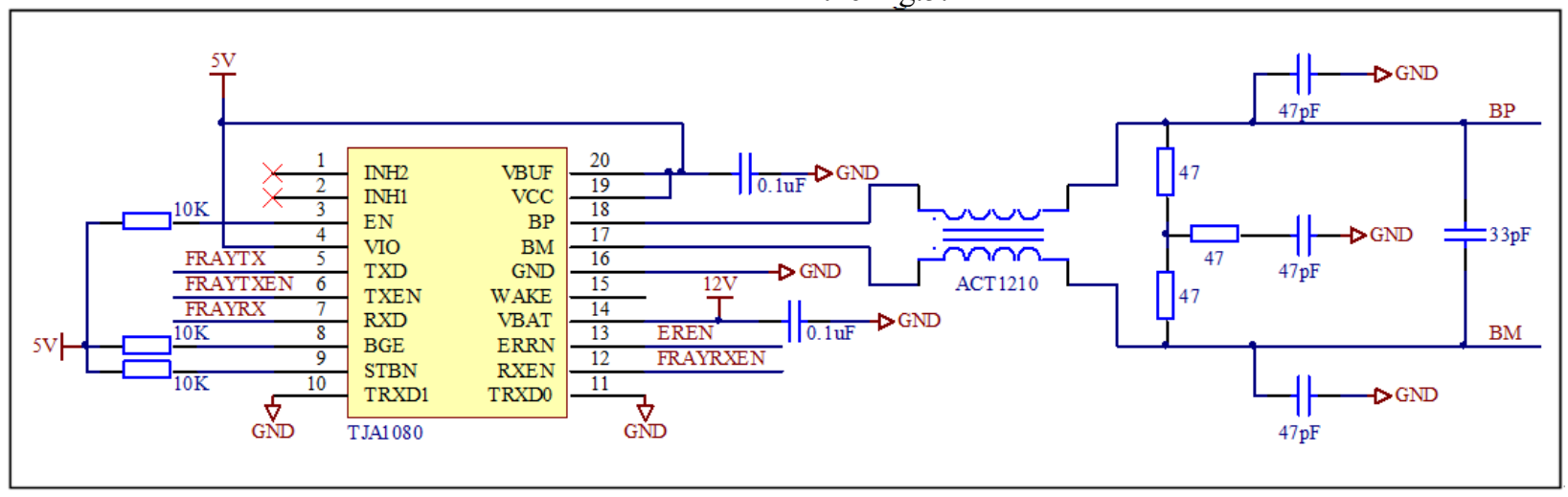

Figure 5. Driving Circuit Schematic.

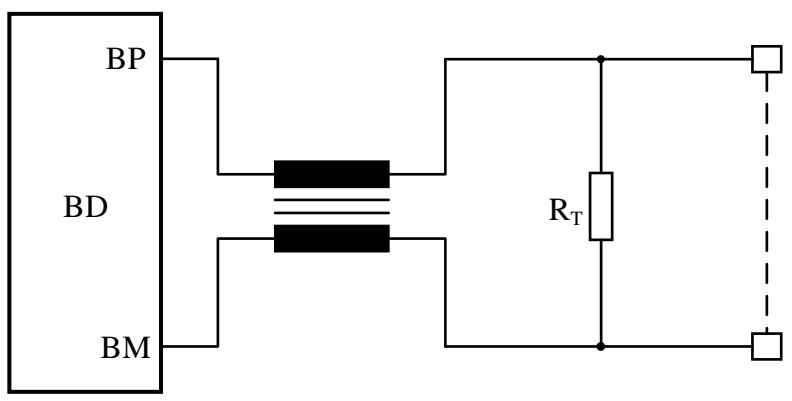

Figure 6. General Termination.

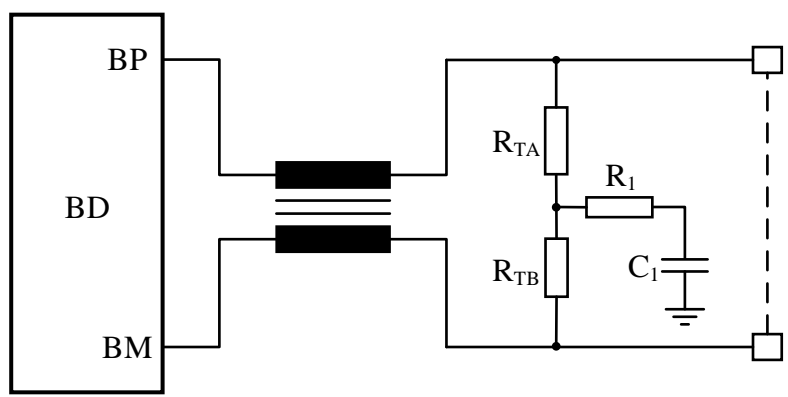

Figure 7. Separation Termination.

This design only requires the node to operate in the normal transceiver mode, then the TRXD0 and the TRXD1 are grounded. The pins 1-2 are used for power management and need to be used with the bus monitor. As there are no FlexRay bus monitor products currently on the market, these two pins are left empty. The pins 5-7 are the interface between TJA1080 and the communication controller for sending and receiving data. The pins 12-13 are used to feed status information back to the MCU. The BP and BM are a pair of differential signal lines, which is similar to $\mathrm{CANH}$ and CANL in the CAN bus.

In general, the simplest method is to terminate a resistor between BP and BM of the bus as shown in Fig.6. In order to better EMC performance, this design adopts the separation termination method, and places the common mode choke between the bus driver and the separation termination resistors as shown in the Fig.7. It divides the resistor in Fig. 6 into two equal parts $\mathrm{R}_{\mathrm{TA}}$ and $\mathrm{R}_{\mathrm{TB}}, \mathrm{R}_{1}-\mathrm{C}_{1}$ series branch is connected between the ground and the common node of the two separate termination resistors, providing a path for the common mode signal. For $\mathrm{R}_{\mathrm{TA}}$ and $\mathrm{R}_{\mathrm{TB}}$, the resistance is as close as possible, and the equal value is put to use universally. The common mode choke has a large impedance to the common mode signal, which can make the current drive capability on the two signal cables consistent, but in the opposite direction.

In addition, it also includes power supply module, serial port module, etc. The main purpose is to make the FlexRay node working properly and easy to debug, and here is not described in detail.

\section{Conclusion}


According to the communication transmission demand of flight control system of pint and medium-sized UAV, this paper designs a FlexRay communication node based on the bus driver TJA1080 as the medium, which can realize the data exchange and sharing in the FlexRay bus. In this bus node design process, it focuses on a number of key points to ensure the real-time and reliability of communication transmission, and it still needs further investigations of FlexRay bus technology in aerospace applications.

\section{References}

1. J Lu, Research on the development of airborne bus technology, Electronic Test, 7:72-73(2017).

2. Y Ding, Review of Airborne Avionics Bus System Development, Avionics Technology, 02:1-7(2003).

3. X Ning, Airborne computer and its bus, National Conference on Signal and Intelligent Information Processing and Application, 54-55(2011).

4. L Li, J Zhang, Comparison of Several Common Buses in Airborne Computers, Aeronautical Computing Technique, 35:103-105(2005).
5. Y Feng, H Dou, Application of CAN Bus in Aviation Field, Advances in Aeronautical Science and Engineering, 02, 231-235(2011).

6. Z Gu, G Han, H Zeng, Q Zhao, Security-Aware Mapping and Scheduling with Hardware CoProcessors for FlexRay-based Distributed Embedded Systems, IEEE Transactions on Parallel \& Distributed Systems, (2016).

7. Kanajan S A J. Sensitivity Analysis on Flexray Dynamic Segment Design Parameters_ International Conference on Systems \& Networks Communications: International Conference on Systems \& Networks Communications, (2009).

8. Y Li, X Chen, C Li, Design and Research of Serial Interface Unit Based on FlexRay Bus Flight Control Computer, Computer Measurement \& Control, 02:94-98(2016).

9. Y Liu, D Cao, X Lv, The flight control computer design of the FlexRay bus, Microcontroller \& Embedded Systems, 04:8-11(2015).

10. Z Liao, Q Gao, D Yuan, Design of FlexRay Node Drive Circuit Based on TJA1080, Journal of Ordnance Equipment Engineering, 10:99-102(2015). 